\title{
Cellular immunotherapy using dendritic cells against multiple myeloma
}

\author{
Thanh-Nhan Nguyen-Pham ${ }^{1,2}$, Youn-Kyung Lee ${ }^{1,3}$, Hyun-Ju Lee ${ }^{1}$, Mi-Hyun Kim ${ }^{1,3}$, Deok-Hwan Yang ${ }^{1,2}$, \\ Hyeoung-Joon Kim², Je-Jung Lee ${ }^{1,2,3}$ \\ ${ }^{1}$ Research Center for Cancer Immunotherapy, ${ }^{2}$ Department of Hematology-Oncology, Chonnam National University Hwasun \\ Hospital, ${ }^{3}$ Vaxcell-Bio Therapeutics, Hwasun, Korea
}

p-ISSN 1738-7949 / e-ISSN 2092-9129

http://dx.doi.org/10.5045/kjh.2012.47.1.17

Korean J Hematol 2012;47:17-27.

Received on December 12, 2011

Revised on February 9, 2012

Accepted on March 2, 2012

*This study was financially supported by grant no. 2011-0005285 from the General Researcher Program Type II of the National Research Foundation of Korea; grant no. RTI05-01-01 from the Regional Technology Innovation Program of the Ministry of Commerce, Industry, and Energy; grant no. A000200058 from the Regional Industrial Technology

Development Program of the Ministry of Knowledge and Economy; grant no. 1120390 from the National R\&D Program for Cancer Control, Ministry for Health and Welfare; and grant no. 2011-0030034 from the Leading Foreign Research Institute Recruitment Program through the National Research Foundation of Korea (NRF), funded by the Ministry of Education, Science, and Technology (MEST), Republic of Korea.

\section{Correspondence to}

Je-Jung Lee, M.D., Ph.D.

Department of Hematology-Oncology, Chonnam National University Hwasun Hospital, 322, Seoyangro, Hwasun 519-763, Korea

Tel: $+82-61-379-7638$

Fax: $+82-61-379-7626$

E-mail: drjejung@chonnam.ac.kr

(C) 2012 Korean Society of Hematology
Cellular therapy with dendritic cells (DCs) is emerging as a useful immunotherapeutic tool to treat multiple myeloma (MM). DC-based idiotype vaccination was recently suggested to induce idiotype-specific immune responses in MM patients. However, the clinical results so far have been largely disappointing, and the clinical effectiveness of such vaccinations in MM still needs to be demonstrated. DC-based therapies against MM may need to be boosted with other sources of tumor-associated antigens, and potent DCs should be recruited to increase the effectiveness of treatment. DCs with both high migratory capacity and high cytokine production are very important for effective DC-based cancer vaccination in order to induce high numbers of Th1-type CD4 ${ }^{+} \mathrm{T}_{\text {cells and } \mathrm{CD}}{ }^{+}$ cytotoxic T lymphocytes. The tumor microenvironment is also important in the regulation of tumor cell growth, proliferation, and the development of therapeutic resistance after treatment. In this review, we discuss how the efficacy of DC vaccination in MM can be improved. In addition, novel treatment strategies that target not only myeloma cells but also the tumor microenvironment are urgently needed to improve treatment outcomes.

Key Words Cellular immunotherapy, Dendritic cell, Multiple myeloma, Cytotoxic T lymphocyte, Immune response

\section{INTRODUCTION}

Multiple myeloma (MM) is a clonal B cell malignant disease that is characterized by the proliferation of plasma cells in the bone marrow (BM) in association with monoclonal protein in the serum and/or urine, immune paresis, skeletal destruction, renal dysfunction, anemia, hypercalcemia, and lytic bone diseases [1]. Although the introduction of high-dose therapy with hematopoietic stem cell trans-

This is an Open Access article distributed under the terms of the Creative Commons Attribution Non-Commercial License (http://creativecommons.org/licenses/by-nc/3.0) which permits unrestricted non-commercial use, distribution, and reproduction in any medium, provided the original work is properly cited. 
plantation (HSCT) and the development of novel molecular targeting agents has resulted in a marked improvement in overall survival, MM still remains incurable [2]. Alternative approaches are clearly needed to prolong both disease-free and overall survival of patients with MM. To prolong the survival of patients with MM who are undergoing allogeneic HSCT, donor lymphocyte infusion can be used successfully as a salvage therapy, based on the graft-versus-myeloma effect in some cases of MM that relapse after allogeneic HSCT $[3,4]$. A clinically significant immune-mediated allogeneic graft-versus-myeloma effect provides the framework for development of immune-based therapeutic options that use antigen-presenting cells (APCs), such as dendritic cells (DCs), in $\mathrm{MM}[4,5]$.

DCs are the most potent APCs for initiating cellular immune responses through the stimulation of naive $\mathrm{T}$ cells. Because of their ability to stimulate T cells, DCs act as links between innate immunity and adaptive immunity in antitumor immune responses [6]. These DCs play a central role in various immunotherapy protocols through generation of cytotoxic T lymphocytes (CTLs) [7]. DC-based vaccines have become the most attractive tool for cancer immunotherapy and have been used in the treatment of more than 20 malignancies [8]. In MM, cellular immunotherapy using DCs is emerging as a useful immunotherapeutic modality [8]. Since tumor antigen-loaded DCs are expected to be able to stimulate tumor-specific CTLs and to overcome T cell tolerance in patients with tumors, the development of DC vaccines that can consistently eliminate minimal residual disease remains an important goal in the field of tumor immunology [9].

\section{IMMUNE DISORDERS IN MM}

Usually, hematologic malignancies elicit weak immunogenic responses that are generally incapable of mediating tumor destruction. They are able to escape immune surveillance via down-regulation of costimulatory molecules and major histocompatibility complex (MHC) molecules as well as the production of immunosuppressive cytokines by tumor cells or by activation of suppressor cells such as regulatory $\mathrm{T}$ cells (Tregs) and myeloid-derived suppressor cells (MDSCs) [10]. In particular, MM induces immune paresis [11]. Patients with MM have DCs that are functionally defective, evidenced by the decreased number of circulating DCs and impaired $\mathrm{T}$ cell stimulatory capacity $[12,13]$. Myeloma cells can produce immuno-inhibitory cytokines, such as interleukin (IL)-10, transforming growth factor beta (TGF- $\beta$ ), vascular endothelial growth factor (VEGF), and IL-6, which play major roles in the pathogenesis of $\mathrm{MM}$, aberrant functions of DCs, and impaired development of effector functions in tumor-specific lymphocytes [12, 13]. In addition, the survival and proliferation of tumor cells are partially facilitated by impaired endogenous immune surveillance against tumor antigens, including the abrogation of DC function, by constitutive activation of the signal transducer and activator of transcription 3 (STAT3) [14]. Impairment in both humoral and cellular immunity in MM is associated with impaired $\mathrm{B}$ cell differentiation and antibody responses [12]; reduced $\mathrm{T}$ cell numbers, specifically $\mathrm{CD}^{+} \mathrm{T}$ cells and abnormal Th1/Th2 $\mathrm{CD}^{+} \mathrm{T}$ cell ratios [15]; impaired CTL responses [16]; and dysfunction of natural killer (NK) cells and natural killer $\mathrm{T}(\mathrm{NKT})$ cells $[17,18]$. In addition, the recruitment and expansion of $\mathrm{CD} 4^{+} \mathrm{CD} 25^{+}$Tregs in $\mathrm{MM}$ patients in the suppression of tumor immunity has been reported [19, 20]. More recently, the discovery of MDSCs revealed these cells to be potent suppressors of tumor immunity and, therefore, a significant impediment to cancer immunotherapy [21]. According to a recent report, the proportion of CD14 $4^{+} \mathrm{HLA}-$ $\mathrm{DR}^{-}{ }^{\text {low }}$ MDSCs and CD4 ${ }^{+} \mathrm{FOXP3}^{+}$Tregs cells was increased in $\mathrm{MM}$ patients at diagnosis and inhibited proliferation of both $\mathrm{CD}^{+}$and $\mathrm{CD}^{+} \mathrm{T}$ cells [22].

\section{CURRENT DC-BASED VACCINES IN MM}

Usually, ex vivo DCs are generated from circulating blood precursors (i.e., monocytes) or BM progenitor cells and are educated with tumor antigens prior to vaccination. $E_{\boldsymbol{X}}$ vivo-generated DCs can be loaded with myeloma-associated antigens as vaccines for patients with MM. The use of immature DCs or mature DCs, method used to induce DC maturation, types of tumor antigens, techniques used to load tumor antigens onto DCs, routes of administration, and dosing schedules are being investigated.

\section{Idiotype-pulsed DCs}

Immunoglobulin idiotype (Id) is a tumor-specific antigen that is produced by each B cell tumor clone. Id protein has been used for immunotherapy in patients with MM $[23,24]$. Id vaccination could induce responses by both antibodies and Id-specific $\mathrm{T}$ cells, including $\mathrm{CD} 4^{+}$and $\mathrm{CD}^{+}$ $\mathrm{T}$ cells, through the presentation of Id protein on the surface of professional APCs. Id-specific CTL lines that kill autologous primary myeloma cells in vitro have been generated. In one report, killing activity was induced by only MHC class I-restriction [25], while another report described both class I- and class II-restriction [26]. Autologous DCs that were generated from MM patients have been shown to efficiently endocytose different classes of Id proteins, and autologous Id-specific CTLs containing both $\mathrm{CD}^{+}$and $\mathrm{CD}^{+} \mathrm{T}$ cells that were generated by Id-pulsed DCs were able to recognize and kill autologous primary myeloma cells in vitro $[26,27]$. Various studies of DC-based Id vaccination in MM have been reported [28-35]. Although Id-specific CTLs and immune responses could be induced in some patients, clinical responses have rarely been observed after vaccination [30], possibly because Id protein is a weak antigen and immature DCs have been used in some studies [28].

\section{Myeloma-associated antigen-loaded DCs}

A variety of myeloma-associated antigens that may induce immune responses from DC-based vaccines have been identi- 
fied in MM patients. Many potential tumor-associated antigens (TAAs) in MM have been investigated, including polymorphic epithelial mucin (MUC1), human telomerase reverse transcriptase (hTERT), preferentially expressed antigen of myeloma (PRAME), Sperm protein 17 (Sp17), Wilms' tumor-I (WTI), Dickkopf-1 (DKK-1), and members of the cancer germ-like family (MAGE, GAGE, BAGE, LAGE, and NY-ESO-1). MUC1 was expressed in all MM cell lines and primary myeloma cells. MUC1-specific CTLs that were induced in vitro using peptide-pulsed DCs or plasma cell RNA-loaded DCs efficiently killed not only target cells pulsed with the antigenic peptide but also MM cells [36, 37]. NY-ESO-1 is the most immunogenic of the cancer testis antigens, proteins that are expressed in a variety of tumors, but whose expression in normal tissue is limited to the testis and placenta [38]. Spontaneous humoral and $\mathrm{CD}^{+} \mathrm{T}$ cell-mediated responses to NY-ESO-1 have been identified in patients with advanced disease [38]. In addition, in vitro monocyte-derived DCs transfected with PTD-NY-ESO-1 protein can induce $\mathrm{CD}^{+}$cellular antitumor immunity superior to that achieved with NY-ESO-1 protein alone [39]. Sp17-specific HLA class I-restricted CTLs were successfully generated by DCs that had been loaded with recombinant Sp17 protein and were able to kill autologous tumor cells that expressed Sp17 [40]. The overexpression of hTERT on MM compared to the expression levels in normal cells indicated that this telomerase also could be used as a myeloma-associated antigen. hTERT was capable of triggering antitumor-CTL responses and killing hTERT ${ }^{+}$tumor cells [41]. Recently, a report demonstrated that activated T lymphocytes were able to successfully kill myeloma cells after stimulation by DCs loaded with hTERT- and MUC1-derived nonapeptides [42]. DKK1, a novel protein that is not expressed in most normal tissues but is expressed in almost all myeloma cells, may be an important antigenic target for antimyeloma immunotherapy. DKK1-specific CTLs that were generated by DCs pulsed with DKK1 peptides were specifically lysed by autologous primary myeloma cells and DKK1-positive cell lines [43]. In general, DCs loaded with TAAs may be promising agents for use in immunotherapy against MM.

\section{Whole tumor antigen-loaded DCs}

Although a single TAA can induce antitumor immune response against $\mathrm{MM}$, tumors may escape immune recognition via down-regulation of specific antigen expression. In contrast, DCs loaded with antigens derived from whole tumor cells can improve the antitumor response, limiting the risk of immunological escape. Numerous reports have described these alternative approaches, such as DCs pulsed with myeloma lysates $[44,45]$, DCs pulsed with myeloma apoptotic bodies [46, 47], DCs transfected with myeloma-derived RNA [37], DCs pulsed with myeloma-derived heat shock protein (HSP) gp96 [48, 49], and DC-myeloma cell hybrids $[50,51]$. These techniques have the advantage of allowing the presentation of multiple epitopes to MHC on DCs, therefore inducing polyclonal $\mathrm{T}$ cell responses from many potentially unknown TAAs and reducing the probability of immune escape by a single TAA. DCs loaded with myeloma cell lysates induced much more potent cytotoxicity in autologous plasma cells than Id protein-pulsed DCs did, suggesting the superiority of the myeloma cell as a source of a tumor antigen [45]. The apoptotic bodies derived from myeloma cells have also been used as tumor antigens for loading onto DCs. Interestingly, these apoptotic bodies were shown to be more effective than cell lysates at inducing CTLs against autologous myeloma cells [52]. HSPs are a class of functionally related proteins whose expression is increased when cells are exposed to elevated temperatures or other stresses. Tumor-derived HSPs, such as HSP70 and gp96, are immunogenic, potent stimulators of tumor-specific CTLs. The specific CTLs that were stimulated by myeloma-derived gp96-loaded DCs were able to lyse myeloma cells but not normal blood cells in a MHC class I-restricted manner [48, 49]. Moreover, fusion of autologous DCs with patient-derived tumors stimulated both helper and cytotoxic $\mathrm{T}$ cell responses through the presentation of internalized and newly synthesized antigens [51]. In mouse MM models, vaccination with DCs fused with either plasmacytoma cells or tumor cells resulted in eradication of tumors and protective immunity against subsequent tumor challenge [50]. In general, the production of DC vaccines using whole tumor antigens has become a promising tool for immunotherapy against MM.

\section{CLINICAL USES OF DC-BASED VACCINES FOR MM}

Trial protocols and responses are summarized in Table 1. Clinical trials of DC-based vaccines for MM have been restricted until now. Almost all of these clinical trials used Id-pulsed DCs alone or in combination with adjuvants such as cytokines or KLH. Recently, a phase I study was undertaken, in which patients with MM were vaccinated with an autologous DC/tumor cell fusion in combination with granulocyte-macrophage colony-stimulating factor (GMCSF) administration [53]. A commercial product is currently being tested in a phase III trial (Mylovenge ${ }^{\mathrm{TM}}$, Dendreon Corp., Seattle, WA, USA). Mylovenge (APC8020) is conducted by pulsing autologous DCs with the patient's Id. A recent report describing this commercial product showed the long-term survival of those receiving the vaccine in comparison with the survival of all other patients with MM who underwent autologous HSCT [54].

\section{HOW DC-BASED VACCINES IN MM CAN BE IMPROVED}

The goal of DC-based vaccines in cancer immunotherapy is to induce the expansion of tumor-specific $\mathrm{T}$ cells and establish immune responses. Cellular immunotherapy using DC-based vaccines have been used as therapeutic agents in MM patients; however, few patients experienced tumor regression, and the most common responses to current DC 
Table 1. Summary of clinical studies using DC-based vaccinations.

\begin{tabular}{|c|c|c|c|c|c|}
\hline Author & DC type & TA & Adjuvant & Immune responses & Clinical responses \\
\hline Liso et al. [29] & imDC & Id & $\pm \mathrm{KLH}$ & $4 / 24$ Id-specific & $17 / 26 \mathrm{SD}$ \\
\hline Lim et al. [28] & imMo-DC & Id & KLH & $\begin{array}{l}5 / 6 \text { Id-specific; } 2 / 6 \text { Id-specific IFN- } \gamma ; 3 / 6 \text { increase } \\
\text { in Id-specific CTL frequency }\end{array}$ & 6/6 PD \\
\hline $\begin{array}{l}\text { Reichardt et al. } \\
\text { [32] }\end{array}$ & imDC & Id & None & 2/12 Id-specific proliferation; $1 / 3$ Id-specific CTL & 2 relapse; $8 / 10$ PD; $2 / 10$ SD \\
\hline Titzer et al. [30] & CD34-DC & Id & None & $\begin{array}{l}\text { 4/10 Id-specific T cell proliferation; } 1 / 10 \\
\text { decreased BM plasmacytosis }\end{array}$ & 1/10 SD; 9/10 PD \\
\hline Cull et al. [89] & imMo-DC & Id & None & $\begin{array}{l}2 / 2 \text { Id-specific T cell proliferation; no Id-specific } \\
\text { CTL response }\end{array}$ & $2 / 2 \mathrm{PD}$ \\
\hline Yi et al. [31] & $\mathrm{mMo}-\mathrm{DC}$ & Id & IL-2 & $\begin{array}{l}2 / 5 \text { Id-specific T cell proliferation; } 5 / 5 \text { Id-specific B } \\
\text { cell proliferation; } 4 / 5 \text { Id-specific IFN- } \gamma\end{array}$ & 1/3 PR; 3/5 SD; $1 / 5 \mathrm{PD}$ \\
\hline $\begin{array}{l}\text { Bendandi et al. } \\
\text { [33] }\end{array}$ & $\mathrm{mMo}-\mathrm{DC}$ & Id & None & $\begin{array}{l}4 / 4 \text { anti-KLH response; } 2 / 4 \text { Th } 1 \text { cytokines } \\
\text { response }\end{array}$ & 1/4 SD; 3/4 PD \\
\hline Lacy et al. [54] & $\begin{array}{l}\text { APC8020 } \\
\text { (Mylovenge) }\end{array}$ & Id & None & None reported & $\begin{array}{l}\text { 6/26 CR; } 2 / 26 \mathrm{PR} ; 19 / 27 \mathrm{SD} \\
\text { Overall survival: } 5.3 \text { years of } \\
\text { follow-up for surviving patients }\end{array}$ \\
\hline Yi et al. [34] & CD40 L-DCs & Id & KLH & $\begin{array}{l}\text { 9/9 Id-specific IFN- } \gamma ; 5 / 9 \text { Id-specific CTL response; } \\
\text { 8/9 anti-KLH response }\end{array}$ & $\begin{array}{l}6 / 9 \text { SD; } 3 / 9 \text { slowly PD } \\
4 / 6 \text { continue SD after } 5 \text { years }\end{array}$ \\
\hline $\begin{array}{l}\text { Rosenblatt et al. } \\
\text { [53] }\end{array}$ & $\begin{array}{l}\text { DC/tumor } \\
\text { fusion }\end{array}$ & & GM-CSF & $\begin{array}{l}\text { 11/15 CD4 and CD8 response with autologous } \\
\text { myeloma cells; } 5 / 5 \text { tested anti-MUC1 response }\end{array}$ & $\begin{array}{l}11 / 16 \text { SD }(3 / 11>1 \text { year } S D ; 8 / 11 \\
2.5-5 \text { month SD) }\end{array}$ \\
\hline Röllig et al. [35] & mMo-DC & Id & $\mathrm{KLH}$ & $\begin{array}{l}\text { 5/9 Id-specific T cell proliferation; } 8 / 9 \text { Id-specific } \\
\text { cytokines response }\end{array}$ & $\begin{array}{l}\text { 3/9 M protein decrease; } 5 / 9 \mathrm{M} \\
\text { protein stable }\end{array}$ \\
\hline
\end{tabular}

Abbreviations: DC, dendritic cell; TA, tumor antigen; imDC, immature DC; Mo-DC, monocyte-derived DC; Id, idiotype; mMo-DC, mature Mo-DC; KLH, keyhole limpet hemocyanin; CTL, cytotoxic T lymphocyte; PD, progressive disease; PR, partial response; SD, stable disease; CR, complete response.

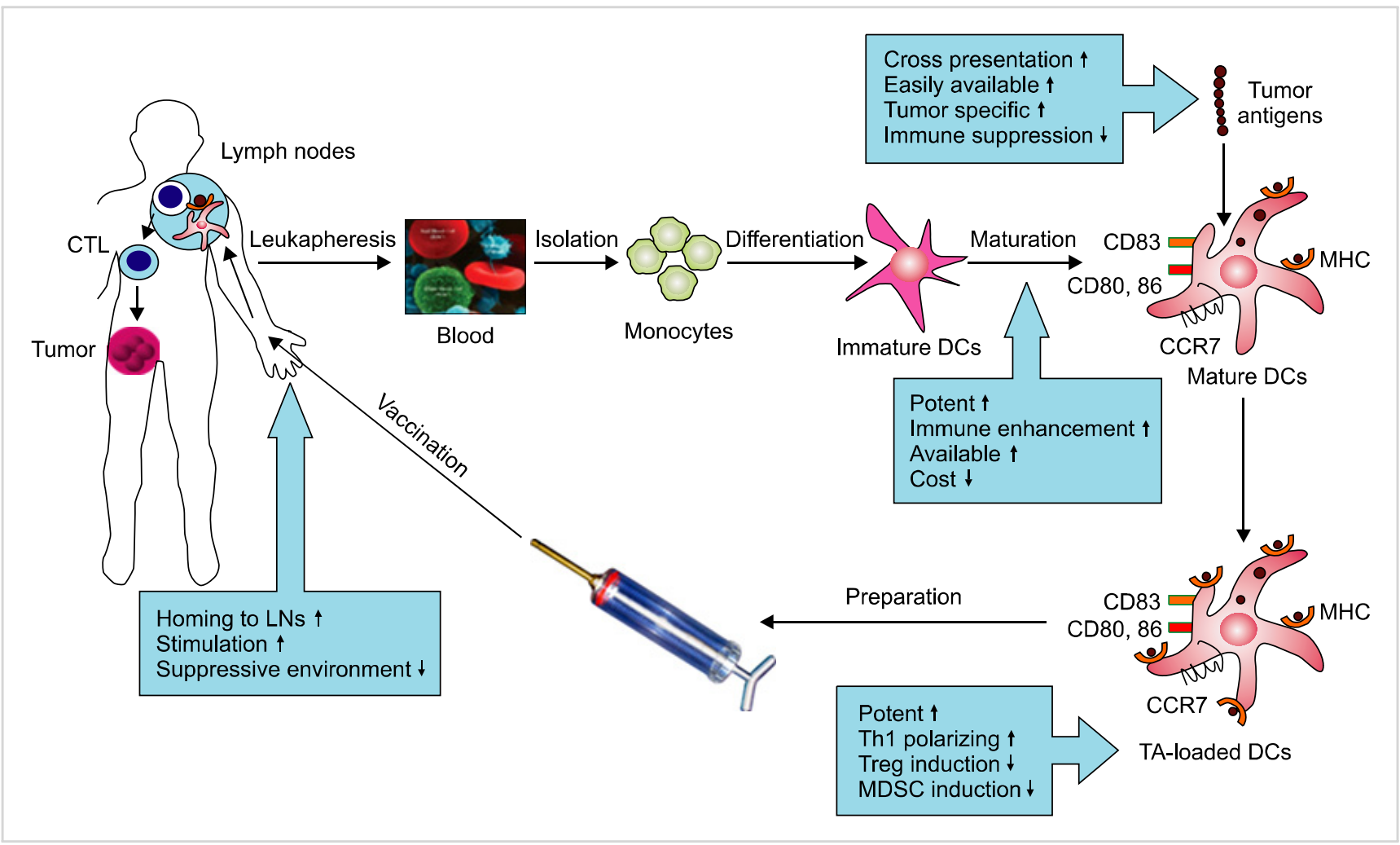

Fig. 1. Key points to improve DC vaccination in cancer patients. Abbreviations: CTL, cytotoxic T lymphocyte; DCs, dendritic cells; TA, tumor antigen; LNs, lymph nodes; Treg, regulatory T cell; MDSC, myeloid-derived suppressor cell. 
vaccines are only antigen-specific immune responses. This is required in a new strategy for the treatment of cancer, in which the intrinsic abilities of the immune system response to the DC-based vaccine has been modified to enhance the efficacy of vaccination. In particular, DCs in MM patients are a target of tumor-associated suppressive factors from the microenvironment, since ex vivo DCs, which are usually generated from patients, are functionally defective, resulting in their suppressed ability to support effector functions in tumor-specific immune responses $[12,13]$. In the generation of DCs, the effects of several immunosuppressive factors in the tumor microenvironment, including the production of inhibitory cytokines, activation of STAT3, expansion of Tregs, and significant suppressive effects of MDSCs, have been investigated. Therefore, the recent focus has been on improving the efficacy of DC vaccines against tumors (Fig. $1)$.

\section{1. $\alpha$-type 1-polarized DCs as highly potent inducers of CTLs against cancers}

The initial success of DC-based vaccines involving immature or partially-mature "first-generation" DCs has been reported $[55,56]$. The first therapeutic DC-based cancer vaccine against prostate cancer (Sipuleucel-T/Provenge) received US FDA approval and demonstrated the ability of the vaccine to prolong overall survival in patients with highly advanced cancer [57]. However, such DCs express suboptimal levels of costimulatory molecules and constitute weaker immunogens than subsequently implemented mature DCs, the "second generation" of clinically applied standard DCs (sDCs). sDC vaccines induced by cytokine cocktails containing IL-1 $\beta / \mathrm{TNF}-\alpha / \mathrm{IL}-6 /$ prostaglandin $\mathrm{E}_{2}\left(\mathrm{PGE}_{2}\right)$ have been developed [58]. Such DCs are fully mature and exhibit increased expression costimulatory molecules and CCR7 and high migratory responsiveness to lymph node-associated chemokines; they have been widely tested in clinical trials. However, to date, sDC vaccines still have limitations, including the mediation of Th2 polarization, promotion of secretion of the immunosuppressive cytokine IL-10 from DCs, inability to induce the Th1-type response (because $\mathrm{PGE}_{2}$ abolishes the secretion of IL-12p70), and high activity of such DCs in activating Tregs $[59,60]$. In addition, the induction of antitumor CTL responses by DC-based vaccines benefits from high expression of costimulatory molecules, CCR7 responsiveness, and migration in response to lymphoid chemokines from mature DCs [61]. At the same time, high IL-12p70 secretion has been shown to dramatically enhance the ability of DCs to induce tumor-specific Th1 cells and CTLs and to promote tumor rejection in therapeutic mouse models [62]. Unfortunately, both first- and second-generation DCs were unable to induce effective CTL responses. Therefore, several investigators, including our group, have tried to develop potent DCs that possess all required features for inducing effective tumor-specific immune responses. We demonstrated the feasibility of inducing mature but "nonexhausted" DCs, by exposing immature DCs to type 1 and type 2 interferons (IFNs) combined with TNF $\alpha$ or Toll-like receptor (TLR) ligands. In particular, $\alpha$-type 1 -polarized DCs ( $\alpha \mathrm{DC} 1 \mathrm{~s})$ that have been induced to mature using an $\alpha \mathrm{DC} 1$-inducing cytokine cocktail containing IL- $1 \beta$, TNF- $\alpha$, IFN- $\alpha$, IFN- $\gamma$, and polyinosinic:polycytidylic acid [poly(I:C)] have been developed to generate strong functional CTLs in several diseases and are on average 20 -fold higher than sDCs [63, 64]. The resulting $\alpha \mathrm{DC} 1 \mathrm{~s}$ showed high expression of several costimulatory molecules or high secretion of IL-12p70, improving the induction of tumor-specific and functional CTLs in comparison with that in sDCs in vitro and in vivo [64-67]. Recently, we successfully generated $\alpha \mathrm{DC} 1$ s from MM patients with high expression of costimulatory molecules, significant production of IL-12p70, and potent generation of myeloma-specific CTLs [46, 47]. Such a novel strategy would provide improved potency of ex vivo-generated DCs for cancer therapy.

2. Natural killer (NK) cells as helpers cells during induction of type- 1 immunity by DCs

The other strategy to induce potent DCs from patients with $\mathrm{MM}$ was the use of helper cells to promote type 1-polarization of DCs. NK cells rapidly migrate to sites of infection and control the immune response in viral infections. Indeed, it has been demonstrated that NK cells play a major immunoregulatory role in the development of protective $\mathrm{T}$ cell-mediated immunity against intracellular pathogens and cancers [68]. Such helper activity of NK cells is at least partially mediated by the functional modulation of DCs. This phenomenon depended on the production of IFN- $\gamma$ and TNF- $\alpha$ by activated NK cells [68] and was associated with enhanced tumor antigen cross-presentation and the induction of Th1 and CTL responses [69-71]. Recent data from our laboratory and other groups has demonstrated that such NK-DC interactions promote the subsequent induction of tumor-specific responses in $\mathrm{CD}^{+}$and $\mathrm{CD}^{+} \mathrm{T}$ cells, allowing NK cells to act as helper cells in the development of type- 1 polarized DCs in responses against cancer [69-71]. Resting NK cells that are activated in the presence of TLR agonists, IL-2, and IFN- $\alpha$ can induce potent DCs with enhanced IL-12p70 production in vitro, generating stronger functional CTLs against myeloma cells than sDCs [71].

\section{Combination of IFN and TLR agonists induce potent type-1 polarized DCs}

We also found that the selected combinations of TLR agonists synergistically triggered a Th1-polarizing capacity through production of high amounts of IL-12p70 [72]. However, the major limitation of this combination was the decreased ability of these cells to migrate into lymph nodes compared to that of conventional DCs. When DCs are activated by individual TLR agonists, such as LPS or poly(I:C), or by a combination of 2 TLR agonists, all cells mature and produce high levels of bioactive IL-12p70 in early phase of maturation and after subsequent stimulation with $\mathrm{T}$ cell-related DC activating signal CD40L. In addition, the phenotyes of these matured DCs were markedly enhanced when a combination of type I and type II IFN was added. 
These combinations of stimuli also regulated the expression of CD38 and CD74, markers related to the full activation of DCs [73, 74]. We demonstrated that at the optimal concentration used to stimulate DCs, the combination of 2 TLR agonists with type I and II IFNs can be used to generate fully mature DCs that have high migratory capacity and can maintain IL-12p70-producing capacity. The regulation of CD38 and CD74 in DCs could in turn enhance the migratory activity of DCs in the presence of a combination of 2 TLR agonists and IFNs [75].

\section{Type-1 immunity induced by DCs maturated using natural products of TLR signaling}

Ursolic acid (URC) is isolated from Uncaria rhynchophylla and phytochemically classified as a triterpene. Triterpene compounds have been identified as a unique class of natural products possessing diverse biological activities. Recently, we reported that URC activates human DCs in a fashion that favors Th1 polarization via the activation of TLR2and/or TLR40 dependent IL-12p70 and induces the production of IFN- $\gamma$ by $\mathrm{CD}^{+}$naïve $\mathrm{T}$ cells [76]. In addition, combination URC and IFN- $\gamma$ enhanced the activation of DCs via promotion of IFN- $\gamma$-induced Th1-cell polarization that was dependent on the activation of IL-12p70 and independent of TLR4 [77]. The potential of natural products to enhance DC maturation and activation has important implications for the use of DCs as cancer vaccines.

\section{Enhancement of cross-presentation of DCs by tumor-asso- ciated antigens}

The clinical responses of DC vaccines using Id-pulsed DCs have been disappointing. The use of TAAs can induce more significant immune responses than Id. Although a single TAA can induce antitumor immune responses against MM, tumors may escape immune recognition via down-regulation of specific antigen expression. In addition, only a small number of tumor samples from MM patients showed a similar level of TAA expression, limiting the usefulness of TAAs in MM. Therefore, to overcome the effects of TAA-based immunotherapy, our group has attempted to use other tumor antigens that improve the cross-presentation of the DC vaccine in patients with MM.

The selected antigen should possess the best characteristics for induction of cross-presentation and should be tumor specific and easily available, but should not induce immune suppression. Whole tumor antigens, including myeloma cell lysates and apoptotic bodies from myeloma cells, are the best tumor antigens and have been selected by many investigators. In practical terms, a number of patients with MM have less than 50\% myeloma cells in the $\mathrm{BM}$ at diagnosis or during progression of the disease. When mononuclear cells from the BM are used as a source of tumor antigens, contamination with normal cells, especially lymphocytes, may occur. Thus, it is necessary to use purified, optimized myeloma cells, if possible, as a source of tumor antigens for the generation of myeloma-specific CTLs stimulated by DCs [44]. We have shown that the function of DCs was affected by the concentration of myeloma cell lysates (i.e., higher concentrations of lysates suppressed $\mathrm{T}$ cell stimulatory capacities) [44]. CTLs that were generated by purified, optimized myeloma cell lysates pulsed with DCs demonstrated much stronger cytotoxicity against autologous plasma cells. These findings indicate that it is important to optimize the concentration of myeloma cell lysates that were loaded onto DCs to potentiate their function.

The use of whole tumor cells instead of single antigens may enhance antitumor effects, but may also target multiple tumor variants and counteract tumor immune evasion. However, it is impractical to obtain sufficient amounts of purified autologous myeloma cells for tumor antigens in the clinical setting of MM. As an alternative source of tumor-relevant antigens, allogeneic tumor cells or established cancer cell lines have been used to overcome this limitation in various tumors [46, 47]. In clinical practice, allogeneic myeloma cell lines may be an effective source of universal tumor antigens that could be used to load DCs for the generation of myeloma-specific CTLs in MM patients. Tumor antigens derived from DC-loaded irradiated allogeneic myeloma cells could generate myeloma-specific CTLs against autologous myeloma cells in patients with MM [46, 71]. The success of using an allogeneic myeloma cell line as tumor antigens led to the possibility that allogeneic myeloma cells could also be used as a viable source of tumor antigens in the context of appropriate major MHC alleles to autologous CTLs. We investigated the possibility of using autologous DCs loaded with an apoptotic allogeneic matched monoclonal immunoglobulin subtype of myeloma and showed that the CTL generated by these tumor antigen-loaded DCs could generate myeloma-specific CTLs against autologous myeloma cells in MM patients [47]. These findings suggested that allogeneic myeloma cell lines and allogeneic matched monoclonal immunoglobulin subtype of myeloma were effective tumor antigens capable of inducing functional CTLs against patients' own myeloma cells.

\section{Blocking immunosuppressive activity during tumor anti- gen loading}

The suppressive effects of tumor cells during DC generation have been explained previously by the ability of the tumor microenvironment to suppress DC differentiation [78]. This suppression is due to the activation of STAT3 and the production of immunosuppressive factors. These factors can influence STAT3 and extracellular signal-regulated kinase (ERK) phosphorylation, resulting in hyperactivation of STAT3 and ERK, which may be responsible for defective DC differentiation [79]. In addition to generate potent and specific tumor antigen-loaded DCs for vaccination, alternative methods have attempted to restore defective DC function and to enhance DC function in MM. The immune-mediated antitumor effects of DCs are enhanced by inhibition of the janus-activated kinase 2 (JAK2)/STAT3 pathway [80], inhibition of p38 or activation of the MEK/ERK or mitogen-activated protein kinase (MAPK) pathways, and neutralization of IL-6 [81]. Recently, we found that when 
MM-specific DCs were generated by loading tumor lysates from myeloma cells collected at diagnosis or during the relapsed/progressive state, these DCs showed lower phenotypic maturation, less T cell stimulatory capacity, less CTL activity, and highly abnormal IL-6 and IL-12 secretion compared to the secretion by unloaded DCs. Moreover, the levels of VEGF, phospho-STAT3, and phospho-ERK1/2 in DCs were significantly higher than in unloaded DCs. After neutralization of VEGF activity, DC functions, signal transduction, and cytokine production returned to normal. Therefore, inhibitory factors and abnormal signaling pathways during maturation with tumor antigens in DCs may be responsible for the defective activity of DCs in MM, and these abnormalities may be overcome by neutralizing the signaling that would lead to a suppressed immune response [82]. Currently, we are working to develop strategies that recover dysfunction of DCs caused from loading tumor antigens through treatment of myeloma cells with a combination of the selective JAK/STAT3 inhibitor JSI-124 and proteasome inhibitor bortezomib. We observed that production of inhibitory cytokines, such as IL-10, IL-23, and especially IL-6, which is known as the main plasma cell growth factor that induces DC dysfunction in MM patients, was down-regulated in DCs loaded with JSI-124- and bortezomib-induced dying myeloma tumor cells. Furthermore, phospho-STAT3 was also down-regulated in these DCs. Consistent with their recovery functions, these DCs displayed a superior ability to induce myeloma-specific responses of CTLs (unpublished data). We found that pretreatment of myeloma cells with a combination of JSI-124 and bortezomib recovered DC dysfunction caused by loading of dying myeloma cells through the up-regulation of Hsp90 and down-regulation of STAT3 phosphorylation and inhibitory cytokine production. These DCs could generate potent myeloma-specific CTLs.

\section{Regulation of the migratory patterns of DCs}

In vitro-generated $\mathrm{DCs}$ that are used for targeting local lymph nodes in vaccination protocols are highly sought after, but difficult to achieve in practice. Type 1-polarized DCs with higher levels of IL-12p70 and potent CTL generation targeting are limited by their migratory capacity to primary lymph nodes due to the relatively lower expression of CCR7 in these DCs than in sDCs. The first important mediator in the mobilization of DCs to lymph nodes is CCR7. However, upregulation of CCR7 alone is insufficient to drive DC migration toward CCL19 and CCL21. Up-regulation of CD38 and down-regulation of CD74 regulate DC migration in vitro and in vivo $[73,74]$. We recently reported that the migratory capacity of DCs is enhanced by the regulation of CD38, CD74, and CCR7 expression on DCs, resulting from the TLR-agonist-mediated synergistic effects of type I and II IFNs on the regulation of DC migration and providing a novel approach to improving vaccination efficacy [75].

The efficacies of 3 features, namely, fully mature status, high migratory responsiveness to lymph-node associated chemokines (CCR7 ligands), and ability to produce high levels of IL-12p70, determine whether DCs are appropriate carriers for anticancer vaccines [63]. However, although $\alpha$-type 1-polarized DCs possess all of the above features, the expression of CCR7 and the migration of DCs in response to the CCR7 ligand (CCL19 and CCL21) were lower in these DCs than in conventional sDCs $[63,64]$ due to the lack of $\mathrm{PGE}_{2}$, a critical component of the standard cytokine cocktail that is important for the expression of CCR7 and the responsiveness of DCs to CCR7 ligands [83]. We also investigated whether migration could be enhanced by increasing the expression of CCR7 or the migration of DCs and found that after stimulation of monocyte-derived DCs by CCL21 $2 \mathrm{hr}$ before the end of maturation by $\alpha$-type 1 -polarized cytokine cocktail, the migration of harvested DCs through transwells was faster than that of unstimulated DCs. Interestingly, phenotypes and cytokine production levels were similar in both DCs. Furthermore, we also found that CCL21treated DCs could induce the ability to stimulate tumorspecific responses of CTLs by the enhancement of CXCL10 (IP-10) secretion (unpublished data). We are now investigating this phenomenon to generate monocyte-derived DCs in $\mathrm{MM}$ patients and in vivo mouse models.

\section{Regulation of the suppressive environment}

Therapeutic DC vaccines need to be highly effective at inducing the expansion of tumor-specific $\mathrm{T}$ cells and need to avoid interaction with and induction of immunosuppressor cells, such as Tregs and MDSCs. Recently, type 1-polarized DCs were shown to suppress the secretion of CCL22 (Tregand Th2-type attracting chemokines), enhance the secretion of CCL5 and CXCL10 (Th1- and effector T cell-attracting chemokines), and suppress the induction of Tregs compared to sDCs [84]. In addition, to enhance the antitumor effects of DC-based vaccines in preclinical in vivo mouse models, we have developed several models of combination therapy using DCs and immunomodulatory drugs, including cyclophosphamide and lenalidomide. Cyclophosphamide is frequently used to enhance or augment antitumor effects in cancer immunotherapy [85]. Low-dose cyclophosphamide may enhance the antitumor efficacy of DC vaccines by increasing the proportion of IFN- $\gamma$ secreting lymphocytes and suppressing the proportion of $\mathrm{CD}^{+} \mathrm{CD} 25^{+} \mathrm{FoxP}^{+}$Tregs in tumor-bearing mice [85]. The result of a clinical trial using allogeneic DC vaccines combined with low-dose cyclophosphamide has revealed that the combination therapy could induce stronger antitumor responses compared to the DC vaccine alone [86]. Recently, we developed a cancer model of combination therapy in mice showing that a single administration of low-dose cyclophosphamide before the first DC vaccination augmented the antitumor effects of DC vaccines to completely eradicate the tumor, prolonging the survival of vaccinated mice [70].

We are developing a mouse myeloma combination therapy model, in which the myeloma tumor-bearing mice were vaccinated with a combination of DCs and lenalidomide. Lenalidomide is a thalidomide analog that is more potent and has less adverse effects [11]. Lenalidomide can induce apoptosis; inhibit the production of cytokines (IL-6, VEGF, 
and TNF- $\alpha$ ) in BM; and stimulate T cell and NK cell proliferation, cytotoxicity, and cytokine (IL-2, IFN- $\gamma$ ) production [11]. In addition, lenalidomide can inhibit the frequency and function of Tregs, resulting in inhibition of Treg expansion and forkehad box P3 (FOXP3) expression in cancer patients [87]. Interestingly, this drug can also induce the activation of APC function, resulting in upregulation of CD40, CD80, and CD86, in chronic lymphocytic leukemia [88]. Therefore, lenalidomide could be used as an immunomodulatory drug in order to enhance immune responses against cancer. Our in vitro study showed that lenalidomide enhanced the maturation and function of DCs in synergistic stimulation with LPS to increase phenotype expression, IL-12p70 production, T cell stimulation capacities, and cytotoxic activities against myeloma cells. In addition, lenalidomide combined with LPS induced type 1-polarization of naïve $\mathrm{T}$ cells and suppressed the generation of Tregs (unpublished data). Moreover, our in vivo mouse myeloma model showed that a treatment combining the immunomodulatory drug lenalidomide with a DCs vaccine improved antitumor immunity by inhibiting immunosuppressor cells and recovering effector cells and inducing superior polarization of the Th1/Th2 balance in favor of the Th1 response. This immunomodulatory effect may be a crucial component of the enhancer-like properties of lenalidomide in the context of antitumor immunity against MM (unpublished data).

\section{FUTURE PERSPECTIVES}

Despite their limitations, recent clinical studies have suggested that DC-based vaccinations may be potential therapies that induce tumor responses and prolong the survival of patients with MM. Further investigations are needed to increase DC-based potency and improve immune responses, and these investigations should focus on the identification of alternative tumor antigens uniquely or specifically expressed on myeloma cells, recovery or restoration of the dysfunction of DCs in MM patients, induction of T cells with desirable effector functions rather than regulatory functions, migration into lymph nodes to stimulate $\mathrm{T}$ cells, and clarification of the ability of tumor-specific CTLs to recognize and kill tumor cells. We expect that type 1-polarized DCs can be developed to generate strong functional myeloma-specific CTLs. Allogeneic myeloma cell lines or allogeneic myeloma cells may be an effective source of universal tumor antigens that could be used to load to DCs for the successful generation of myeloma-specific CTLs. Eventually, combination therapy, in which a DC vaccine is combined with either alternative therapy, including chemotherapy, radiation therapy, molecular target therapy, or other immunotherapy (adoptive T cell therapy, NK cell therapy, etc.), or with an adjuvant, will provide vigorous and maintained immune responses with improved clinical efficacy.

\section{REFERENCES}

1. Kyle RA, Rajkumar SV. Multiple myeloma. N Engl J Med 2004;351:1860-73.

2. Attal M, Harousseau JL. The role of high-dose therapy with autologous stem cell support in the era of novel agents. Semin Hematol 2009;46:127-32.

3. Perez-Simón JA, Martino R, Alegre A, et al. Chronic but not acute graft-versus-host disease improves outcome in multiple myeloma patients after non-myeloablative allogeneic transplantation. Br J Haematol 2003;121:104-8.

4. Harrison SJ, Cook G, Nibbs RJ, Prince HM. Immunotherapy of multiple myeloma: the start of a long and tortuous journey. Expert Rev Anticancer Ther 2006;6:1769-85.

5. Tricot G, Vesole DH, Jagannath S, Hilton J, Munshi N, Barlogie B. Graft-versus-myeloma effect: proof of principle. Blood 1996; 87:1196-8.

6. Banchereau J, Steinman RM. Dendritic cells and the control of immunity. Nature 1998;392:245-52.

7. Reid DC. Dendritic cells and immunotherapy for malignant disease. Br J Haematol 2001;112:874-87.

8. Ridgway D. The first 1000 dendritic cell vaccinees. Cancer Invest 2003;21:873-86.

9. Banchereau J, Palucka AK. Dendritic cells as therapeutic vaccines against cancer. Nat Rev Immunol 2005;5:296-306.

10. Kim R, Emi M, Tanabe K. Cancer immunoediting from immune surveillance to immune escape. Immunology 2007;121:1-14.

11. Quach H, Ritchie D, Stewart AK, et al. Mechanism of action of immunomodulatory drugs (IMiDS) in multiple myeloma. Leukemia 2010;24:22-32.

12. Brown RD, Pope B, Murray A, et al. Dendritic cells from patients with myeloma are numerically normal but functionally defective as they fail to up-regulate CD80 (B7-1) expression after huCD40LT stimulation because of inhibition by transforming growth factorbeta1 and interleukin-10. Blood 2001;98:2992-8.

13. Ratta M, Fagnoni F, Curti A, et al. Dendritic cells are functionally defective in multiple myeloma: the role of interleukin-6. Blood 2002;100:230-7.

14. Yu H, Kortylewski M, Pardoll D. Crosstalk between cancer and immune cells: role of STAT3 in the tumour microenvironment. Nat Rev Immunol 2007;7:41-51.

15. Ogawara H, Handa H, Yamazaki T, et al. High Th1/Th2 ratio in patients with multiple myeloma. Leuk Res 2005;29:135-40.

16. Maecker B, Anderson KS, von Bergwelt-Baildon MS, et al. Viral antigen-specific CD8+ T-cell responses are impaired in multiple myeloma. Br J Haematol 2003;121:842-8.

17. Dhodapkar MV, Geller MD, Chang DH, et al. A reversible defect in natural killer $\mathrm{T}$ cell function characterizes the progression of premalignant to malignant multiple myeloma. J Exp Med 2003;197:1667-76.

18. Jarahian M, Watzl C, Issa Y, Altevogt P, Momburg F. Blockade of natural killer cell-mediated lysis by NCAM140 expressed on tumor cells. Int J Cancer 2007;120:2625-34.

19. Prabhala RH, Neri P, Bae JE, et al. Dysfunctional T regulatory cells in multiple myeloma. Blood 2006;107:301-4.

20. Banerjee DK, Dhodapkar MV, Matayeva E, Steinman RM, 
Dhodapkar KM. Expansion of FOXP3 high regulatory T cells by human dendritic cells (DCs) in vitro and after injection of cytokine-matured DCs in myeloma patients. Blood 2006;108: 2655-61.

21. Ostrand-Rosenberg S, Sinha P. Myeloid-derived suppressor cells: linking inflammation and cancer. J Immunol 2009;182:4499-506.

22. Brimnes MK, Vangsted AJ, Knudsen LM, et al. Increased level of both CD4+FOXP3+ regulatory T cells and CD14+HLA-DR $/$ low myeloid-derived suppressor cells and decreased level of dendritic cells in patients with multiple myeloma. Scand J Immunol 2010;72:540-7.

23. Kwak LW, Taub DD, Duffey PL, et al. Transfer of myeloma idiotype-specific immunity from an actively immunised marrow donor. Lancet 1995;345:1016-20.

24. Bergenbrant S, Yi Q Osterborg A, et al. Modulation of antiidiotypic immune response by immunization with the autologous $\mathrm{M}$-component protein in multiple myeloma patients. $\mathrm{Br} \mathrm{J}$ Haematol 1996;92:840-6.

25. Li Y, Bendandi M, Deng Y, et al. Tumor-specific recognition of human myeloma cells by idiotype-induced CD8(+) T cells. Blood 2000;96:2828-33.

26. Wen YJ, Barlogie B, Yi Q. Idiotype-specific cytotoxic $T$ lymphocytes in multiple myeloma: evidence for their capacity to lyse autologous primary tumor cells. Blood 2001;97:1750-5.

27. Butch AW, Kelly KA, Munshi NC. Dendritic cells derived from multiple myeloma patients efficiently internalize different classes of myeloma protein. Exp Hematol 2001;29:85-92.

28. Lim SH, Bailey-Wood R. Idiotypic protein-pulsed dendritic cell vaccination in multiple myeloma. Int J Cancer 1999;83:215-22.

29. Liso A, Stockerl-Goldstein KE, Auffermann-Gretzinger S, et al. Idiotype vaccination using dendritic cells after autologous peripheral blood progenitor cell transplantation for multiple myeloma. Biol Blood Marrow Transplant 2000;6:621-7.

30. Titzer S, Christensen O, Manzke O, et al. Vaccination of multiple myeloma patients with idiotype-pulsed dendritic cells: immunological and clinical aspects. Br J Haematol 2000;108:805-16.

31. Yi Q Desikan R, Barlogie B, Munshi N. Optimizing dendritic cell-based immunotherapy in multiple myeloma. Br J Haematol 2002;117:297-305.

32. Reichardt VL, Milazzo C, Brugger W, Einsele H, Kanz L, Brossart P. Idiotype vaccination of multiple myeloma patients using monocyte-derived dendritic cells. Haematologica 2003;88:113949.

33. Bendandi M, Rodríguez-Calvillo M, Inogés $\mathrm{S}$, et al. Combined vaccination with idiotype-pulsed allogeneic dendritic cells and soluble protein idiotype for multiple myeloma patients relapsing after reduced-intensity conditioning allogeneic stem cell transplantation. Leuk Lymphoma 2006;47:29-37.

34. Yi Q, Szmania S, Freeman J, et al. Optimizing dendritic cell-based immunotherapy in multiple myeloma: intranodal injections of idiotype-pulsed CD40 ligand-matured vaccines led to induction of type- 1 and cytotoxic T-cell immune responses in patients. $\mathrm{Br}$ J Haematol 2010;150:554-64.

35. Röllig C, Schmidt C, Bornhäuser M, Ehninger G, Schmitz M, Auffermann-Gretzinger S. Induction of cellular immune responses in patients with stage-I multiple myeloma after vaccination with autologous idiotype-pulsed dendritic cells. J Immun- other 2011;34:100-6.

36. Brossart P, Schneider A, Dill P, et al. The epithelial tumor antigen MUC1 is expressed in hematological malignancies and is recognized by MUC1-specific cytotoxic T-lymphocytes. Cancer Res 2001;61:6846-50.

37. Milazzo C, Reichardt VL, Müller MR, Grünebach F, Brossart P. Induction of myeloma-specific cytotoxic T cells using dendritic cells transfected with tumor-derived RNA. Blood 2003;101:97782.

38. Szmania S, Tricot G, van Rhee F. NY-ESO-1 immunotherapy for multiple myeloma. Leuk Lymphoma 2006;47:2037-48.

39. Batchu RB, Moreno AM, Szmania SM, et al. Protein transduction of dendritic cells for NY-ESO-1-based immunotherapy of myeloma. Cancer Res 2005;65:10041-9.

40. Chiriva-Internati M, Wang Z, Salati E, Bumm K, Barlogie B, Lim SH. Sperm protein 17 (Sp17) is a suitable target for immunotherapy of multiple myeloma. Blood 2002;100:961-5.

41. Vonderheide RH, Hahn WC, Schultze JL, Nadler LM. The telomerase catalytic subunit is a widely expressed tumor-associated antigen recognized by cytotoxic $\mathrm{T}$ lymphocytes. Immunity 1999;10:673-9.

42. Ocadlikova D, Kryukov F, Mollova K, et al. Generation of myeloma-specific T cells using dendritic cells loaded with MUC1and hTERT- drived nonapeptides or myeloma cell apoptotic bodies. Neoplasma 2010;57:455-64.

43. Qian J, Xie J, Hong S, et al. Dickkopf-1 (DKK1) is a widely expressed and potent tumor-associated antigen in multiple myeloma. Blood 2007;110:1587-94.

44. Lee JJ, Choi BH, Kang HK, et al. Induction of multiple myeloma-specific cytotoxic $\mathrm{T}$ lymphocyte stimulation by dendritic cell pulsing with purified and optimized myeloma cell lysates. Leuk Lymphoma 2007;48:2022-31.

45. Wen YJ, Min R, Tricot G, Barlogie B, Yi Q. Tumor lysate-specific cytotoxic $\mathrm{T}$ lymphocytes in multiple myeloma: promising effector cells for immunotherapy. Blood 2002;99:3280-5.

46. Yang DH, Kim MH, Hong CY, et al. Alpha-type 1-polarized dendritic cells loaded with apoptotic allogeneic myeloma cell line induce strong CTL responses against autologous myeloma cells. Ann Hematol 2010;89:795-801.

47. Yang DH, Kim MH, Lee YK, et al. Successful cross-presentation of allogeneic myeloma cells by autologous alpha-type 1-polarized dendritic cells as an effective tumor antigen in myeloma patients with matched monoclonal immunoglobulins. Ann Hematol 2011;90:1419-26.

48. Qian J, Wang S, Yang J, et al. Targeting heat shock proteins for immunotherapy in multiple myeloma: generation of myelomaspecific CTLs using dendritic cells pulsed with tumor-derived gp96. Clin Cancer Res 2005;11:8808-15.

49. Qian J, Hong S, Wang S, et al. Myeloma cell line-derived, pooled heat shock proteins as a universal vaccine for immunotherapy of multiple myeloma. Blood 2009;114:3880-9.

50. Hao S, Bi X, Xu S, et al. Enhanced antitumor immunity derived from a novel vaccine of fusion hybrid between dendritic and engineered myeloma cells. Exp Oncol 2004;26:300-6.

51. Vasir B, Borges V, Wu Z, et al. Fusion of dendritic cells with multiple myeloma cells results in maturation and enhanced antigen presentation. Br J Haematol 2005;129:687-700. 
52. Hayashi T, Hideshima T, Akiyama M, et al. Ex vivo induction of multiple myeloma-specific cytotoxic $\mathrm{T}$ lymphocytes. Blood 2003;102:1435-42.

53. Rosenblatt J, Vasir B, Uhl L, et al. Vaccination with dendritic cell/tumor fusion cells results in cellular and humoral antitumor immune responses in patients with multiple myeloma. Blood 2011;117:393-402.

54. Lacy MQ Mandrekar S, Dispenzieri A, et al. Idiotype-pulsed antigen-presenting cells following autologous transplantation for multiple myeloma may be associated with prolonged survival. Am J Hematol 2009;84:799-802.

55. Hsu FJ, Benike C, Fagnoni F, et al. Vaccination of patients with B-cell lymphoma using autologous antigen-pulsed dendritic cells. Nat Med 1996;2:52-8.

56. Nestle FO, Alijagic S, Gilliet M, et al. Vaccination of melanoma patients with peptide- or tumor lysate-pulsed dendritic cells. Nat Med 1998;4:328-32.

57. Madan RA, Gulley JL. Sipuleucel-T: harbinger of a new age of therapeutics for prostate cancer. Expert Rev Vaccines 2011;10: 141-50.

58. Jonuleit H, Kühn U, Müller G, et al. Pro-inflammatory cytokines and prostaglandins induce maturation of potent immunostimulatory dendritic cells under fetal calf serum-free conditions. Eur J Immunol 1997;27:3135-42.

59. Kalinski P, Vieira PL, Schuitemaker JH, de Jong EC, Kapsenberg ML. Prostaglandin E(2) is a selective inducer of interleukin-12 p40 (IL-12p40) production and an inhibitor of bioactive IL-12p70 heterodimer. Blood 2001;97:3466-9.

60. Yamazaki S, Inaba K, Tarbell KV, Steinman RM. Dendritic cells expand antigen-specific Foxp3+ CD25+ CD4+ regulatory T cells including suppressors of alloreactivity. Immunol Rev 2006;212: 314-29.

61. de Vries IJ, Lesterhuis WJ, Scharenborg NM, et al. Maturation of dendritic cells is a prerequisite for inducing immune responses in advanced melanoma patients. Clin Cancer Res 2003;9:5091-100.

62. Okada N, Iiyama S, Okada Y, et al. Immunological properties and vaccine efficacy of murine dendritic cells simultaneously expressing melanoma-associated antigen and interleukin-12. Cancer Gene Ther 2005;12:72-83.

63. Mailliard RB, Wankowicz-Kalinska A, Cai Q et al. Alpha-type-1 polarized dendritic cells: a novel immunization tool with optimized CTL-inducing activity. Cancer Res 2004;64:5934-7.

64. Lee JJ, Foon KA, Mailliard RB, Muthuswamy R, Kalinski P. Type 1-polarized dendritic cells loaded with autologous tumor are a potent immunogen against chronic lymphocytic leukemia. J Leukoc Biol 2008;84:319-25.

65. Wesa A, Kalinski P, Kirkwood JM, Tatsumi T, Storkus WJ. Polarized type-1 dendritic cells (DC1) producing high levels of IL-12 family members rescue patient TH1-type antimelanoma CD4+ T cell responses in vitro. J Immunother 2007;30:75-82.

66. Giermasz AS, Urban JA, Nakamura Y, et al. Type-1 polarized dendritic cells primed for high IL-12 production show enhanced activity as cancer vaccines. Cancer Immunol Immunother 2009; 58:1329-36.

67. Okada H, Kalinski P, Ueda R, et al. Induction of CD8+ T-cell responses against novel glioma-associated antigen peptides and clinical activity by vaccinations with alpha\}-type 1 polarized dendritic cells and polyinosinic-polycytidylic acid stabilized by lysine and carboxymethylcellulose in patients with recurrent malignant glioma. J Clin Oncol 2011;29:330-6.

68. Gerosa F, Baldani-Guerra B, Nisii C, Marchesini V, Carra G, Trinchieri G. Reciprocal activating interaction between natural killer cells and dendritic cells. J Exp Med 2002;195:327-33.

69. Mailliard RB, Son YI, Redlinger R, et al. Dendritic cells mediate NK cell help for Th1 and CTL responses: two-signal requirement for the induction of NK cell helper function. J Immunol 2003;171:2366-73.

70. Pham TN, Hong CY, Min JJ, et al. Enhancement of antitumor effect using dendritic cells activated with natural killer cells in the presence of Toll-like receptor agonist. Exp Mol Med 2010;42: 407-19.

71. Nguyen-Pham TN, Im CM, Nguyen TA, et al. Induction of myeloma-specific cytotoxic $\mathrm{T}$ lymphocytes responses by natural killer cells stimulated-dendritic cells in patients with multiple myeloma. Leuk Res 2011;35:1241-7.

72. Napolitani G, Rinaldi A, Bertoni F, Sallusto F, Lanzavecchia A. Selected toll-like receptor agonist combinations synergistically trigger a $T$ helper type 1-polarizing program in dendritic cells. Nat Immunol 2005;6:769-76.

73. Frasca L, Fedele G, Deaglio S, et al. CD38 orchestrates migration, survival, and Th1 immune response of human mature dendritic cells. Blood 2006;107:2392-9.

74. Faure-André G, Vargas P, Yuseff MI, et al. Regulation of dendritic cell migration by CD74, the MHC class II-associated invariant chain. Science 2008;322:1705-10.

75. Nguyen-Pham TN, Lim MS, Nguyen TA, et al. Type I and II interferons enhance dendritic cell maturation and migration capacity by regulating CD38 and CD74 that have synergistic effects with TLR agonists. Cell Mol Immunol 2011;8:341-7.

76. Jung TY, Pham TN, Umeyama A, et al. Ursolic acid isolated from Uncaria rhynchophylla activates human dendritic cells via TLR2 and/or TLR4 and induces the production of IFN-gamma by CD4+ naive T cells. Eur J Pharmacol 2010;643:297-303.

77. Bae WK, Umeyama A, Chung IJ, Lee JJ, Takei M. Uncarinic acid $\mathrm{C}$ plus IFN- $\gamma$ generates monocyte-derived dendritic cells and induces a potent Th1 polarization with capacity to migrate. Cell Immunol 2010;266:104-10.

78. Savill J, Dransfield I, Gregory C, Haslett C. A blast from the past: clearance of apoptotic cells regulates immune responses. Nat Rev Immunol 2002;2:965-75.

79. Kitamura H, Kamon H, Sawa S, et al. IL-6-STAT3 controls intracellular MHC class II alphabeta dimer level through cathepsin S activity in dendritic cells. Immunity 2005;23:491-502.

80. Nefedova Y, Gabrilovich DI. Targeting of Jak/STAT pathway in antigen presenting cells in cancer. Curr Cancer Drug Targets 2007;7:71-7.

81. Wang S, Hong S, Yang J, et al. Optimizing immunotherapy in multiple myeloma: Restoring the function of patients' monocytederived dendritic cells by inhibiting p38 or activating MEK/ERK MAPK and neutralizing interleukin-6 in progenitor cells. Blood 2006;108:4071-7.

82. Yang DH, Park JS, Jin CJ, et al. The dysfunction and abnormal signaling pathway of dendritic cells loaded by tumor antigen can be overcome by neutralizing VEGF in multiple myeloma. Leuk 
Res 2009;33:665-70.

83. Scandella E, Men Y, Gillessen S, Förster R, Groettrup M. Prostaglandin E2 is a key factor for CCR7 surface expression and migration of monocyte-derived dendritic cells. Blood 2002;100: 1354-61.

84. Muthuswamy R, Urban J, Lee JJ, Reinhart TA, Bartlett D, Kalinski P. Ability of mature dendritic cells to interact with regulatory $\mathrm{T}$ cells is imprinted during maturation. Cancer Res 2008;68:5972-8.

85. Liu JY, Wu Y, Zhang XS, et al. Single administration of low dose cyclophosphamide augments the antitumor effect of dendritic cell vaccine. Cancer Immunol Immunother 2007;56:1597-604.

86. Höltl L, Ramoner R, Zelle-Rieser C, et al. Allogeneic dendritic cell vaccination against metastatic renal cell carcinoma with or without cyclophosphamide. Cancer Immunol Immunother 2005;54:663-70.

87. Galustian C, Meyer B, Labarthe MC, et al. The anti-cancer agents lenalidomide and pomalidomide inhibit the proliferation and function of $\mathrm{T}$ regulatory cells. Cancer Immunol Immunother 2009;58:1033-45.

88. Ramsay AG, Gribben JG. Immune dysfunction in chronic lymphocytic leukemia $\mathrm{T}$ cells and lenalidomide as an immunomodulatory drug. Haematologica 2009;94:1198-202.

89. Cull G, Durrant L, Stainer C, Haynes A, Russell N. Generation of anti-idiotype immune responses following vaccination with idiotype-protein pulsed dendritic cells in myeloma. Br J Haematol 1999;107:648-55. 